\title{
Exemplarität und die Ordnung des Erzieherischen
}

\section{Exemplarität als Thema}

Exemplarität ist kein Wort, das uns heute in der Wissenschaft auf Schritt und Tritt begegnet. Es scheint eher ein hapax legomenon der Wissenschaftssprache zu werden. Ein Blick in einschlägige Handbücher und Lexika erweckt zumindest diesen Eindruck. ${ }^{1}$ Es sieht prima facie so aus, als ob "es sich um einen vernachlässigbaren, nur noch historisch interessanten Begriff handelt «. ${ }^{2}$ Beobachten können wir auch, dass, bis auf wenige Ausnahmen wie z.B. in der Literaturwissenschaft, Exemplarität kaum thematisiert wird, dass von den semantisch verwandten Ausdrücken aber zum Teil rege Gebrauch gemacht wird. ${ }^{3}$ Es sieht auf den zweiten Blick daher so aus, als hätten wir es mit einem Begriff zu tun, der in der Breite als bekannt vorausgesetzt wird. Und in der Pädagogik?

Dort ist zu hören, dass die Diskussion um das exemplarische Lehren und Lernen »ihren Höhepunkt 1945-1965 « hatte. ${ }^{4}$ Seitdem die Differenz von Didaktik und Methode in den Fachdidaktiken verblasst ist und diese sich auf die Seite der Kompetenzforschung geschlagen haben, scheint auch die Schulpädagogik, die vormalige Domäne des pädagogischen Denkens über Exemplarität, das Interesse an diesem Thema verloren zu haben. ${ }^{5}$ Generell kann man festhalten, dass Exemplarität kein
Thema ist, dass in der Pädagogik gegenwärtig viel Beachtung erfährt.

Man mag sich fragen, warum es dazu gekommen ist. Ist ,Exemplarität‘ womöglich weniger wichtig als lebenslanges Lernen، oder semployability`? Dann wäre 'Exemplarität` wohl zurecht aus dem Fokus der Aufmerksamkeit geraten. Vielleicht aber ist das Verschwinden des Themas von der pädagogischen Agenda auch ein Hinweis, dass man in einer anderen Richtung nach einer Erklärung suchen sollte. Vielleicht nämlich ist das Verschwinden Ausdruck für ein Verblassen des Schwerpunktes, unter dem Sachverhalte wie Erziehung, Unterricht und Bildung bisher behandelt worden sind.

Dieser Schwerpunkt war bis zur zweiten Hälfte des 20. Jahrhunderts der Mensch unter dem Gesichtspunkt von Erziehung und Bildung, der insbesondere durch einen allgemeinpädagogisch und -didaktisch reflektierten Unterricht in seinem Aufwachsen unterstützt werden sollte. Auf ihn war der "Grundgedanke « der Exemplarität ausgerichtet. ${ }^{6}$ Der einzelne Mensch war es, der vor allem, aber nicht nur als Schüler "an wenigen Beispielen grundlegende Einsichten « in die allgemeine Verfasstheit der Welt und die regionalen Besonderheiten von Gesellschaften gewinnen sollte. Aus dem Grunde zählt Exemplarität bis heute noch zu den zentralen Prinzipien des pädagogischen Nachdenkens über Erziehung, 
Unterricht und Bildung, die "gewährleistet « sein müssen. ${ }^{7}$ Lehrer sind "für die Bereitstellung von didaktisch-methodisch durchdachten Unterrichtsarrangements verantwortlich, die ein exemplarisches und strukturiertes Lernen an geeigneten Beispielen ermöglichen «. ${ }^{8}$

Diese Aufgabe sollte dabei nicht auf den psychologischen Hinweis reduziert werden, dass Menschen 'Kategorien، erwerben, in denen sie "Objekte oder Ereignisse auf der Basis von Gemeinsamkeiten zu Klassen « zusammenfassen, die sie zur Orientierung in der Welt einsetzen. ${ }^{9}$ Die Exemplarität eines Unterrichtsinhalts muss vielmehr durch eine spezifische Form des Miteinanderumgehens, den erziehenden Unterricht, gemeinsam erarbeitet werden. ${ }^{10}$ Sie gibt sich nicht von sich aus zu erkennen, sie folgt keinem Automatismus, sondern sie muss durch "Abstrahieren und Rückbeziehen « in lehrender Unterstützung erkannt und verstanden werden. ${ }^{11}$

Dreh- und Angelpunkt der Beschäftigung mit Exemplarität ist unter diesem Schwerpunkt die Einübung von Urteilskraft, die nach Immanuel Kant darin besteht, die Differenz Besonderes | Allgemeines situationsangemessen zu handhaben. Vorausgesetzt wurde dazu ein gesellschaftlicher Zusammenhang, relativ zu dem Urteile als möglich angesehen wurden und ihnen die Aufgabe aufgebürdet wurde, den Zusammenhalt der Gesellschaft nicht nur abzubilden, sondern auch zu befördern. Plakativ gesagt: Unter dieser Schwerpunktsetzung stand der Mensch als urteilsbegabtes und -fähiges Mitglied einer diskutierenden Öffentlichkeit im Fokus der Aufmerksamkeit.

All das galt und gilt bis heute generell für das Aufwachsen unter den Bedingungen von Erziehung, Unterricht und Bildung, soweit an diesem Schwerpunkt festgehalten wird. Wer anders als der einzelne Mensch sollte für diese Arbeit angesprochen werden und wer anders sollte die Ernte aus dieser Arbeit einfahren?
Im Zuge der verschiedentlich beschriebenen Versozialwissenschaftlichung des Faches, die zur Etablierung von Kompetenz- und Qualifikationsforschung geführt hat, ist ein neuer Schwerpunkt etabliert worden. Das >Bildungssystem‘, gegliedert nach Subsystemen wie 'Kindergarten', 'Schule،, 'Berufsausbildung usw. und geordnet unter Ausbildungs- und Karrierekriterien, bildet seither den Gegenstandsraum, auf den sich der Großteil der Forschung konzentriert. Sie beschreibt sich selbst unter dem Paradigma einer datenproduzierenden und Steuerungswissen generierenden Bildungsforschung als Beobachterin des 'Bildungssystems ‘. ${ }^{12}$ Von dem Schwerpunkt früherer Tage distanziert sie sich und überlässt ihn der Pädagogik, die offenkundig snäher am Geschehen ist oder den Teilen der Erziehungswissenschaft, die noch nicht ganz auf das Wissenschaftsverständnis der Bildungsforschung umgeschwenkt sind.

Die ,Nähe، der Pädagogik zum erzieherischen Geschehen wird von der Bildungsforschung in der Regel ablehnend beschrieben. Man will nicht im Schlamm des Erziehungsalltags wühlen. Die Bildungsforschung zieht dazu eine klare Grenze: Wer Sachverhalte wie Schule und Unterricht pädagogisch zum Thema macht, äußert Gedanken zu dem, was Erziehung, Bildung und deren institutionelle Organisation aus Sicht der Menschen ausmachen und für sie bedeuten. Um beschreiben zu können, was es für Menschen bedeutet, im Bildungssystem aufzuwachsen und den Erwartungen der anderen ausgesetzt zu sein, mit denen sie umzugehen lernen sollen, werden Annahmen über akzeptable Menschenbilder, Bildsamkeit, ethische Grenzziehungen, tolerable Spielräume, die pädagogischen Zugriffen verschlossen bleiben (sollten), und anderes mehr benötigt. Die Pädagogik liefert problemorientiertes Wissen in diesen Zusammenhängen. Dafür sind Festlegungen, z.B. 
hinsichtlich des Menschenbildes, des Gesellschaftsverständnisses und der Formen des Umgangs, unvermeidlich. Aus dem Grunde steht die Pädagogik unter Ideologieverdacht. Sie engagiert sich, sagt die Kritik, anstatt das Geschehen aus nüchterner Distanz zu beobachten. ${ }^{13}$

Dieser Vorwurf ist schwerwiegend und sollte nicht auf die leichte Schulter genommen werden. Ebenso wichtig ist es aber auch, sich dem Vorwurf nicht einfach zu ergeben. Es sollte vielmehr versucht werden, argumentativ auszuloten, wie der Gegenstand eine gewisse ,Nähe، der Wissenschaft nötig macht und wie die reflexive Distanz zum Geschehen gestaltet werden sollte. Pauschalablehnungen und -zuweisungen von Verantwortung sind da kaum hilfreich. Wir werden deshalb an Günther Bucks Überlegungen zur Exemplarität ansetzen, um zu zeigen, dass man snah، am Gegenstand sein kann, ohne diese 'Nähe، ideologisch zu begründen. In seiner "Methodenlehre der moralischen Bildung " hat er einen Versuch vorgelegt, wie man eine Ordnung des Erzieherischen denken kann, ohne festgefügte Schemata vorzuschreiben. Bucks Überlegungen weisen in eine Richtung, die, konsequent beschritten, die Frage nach dem Status des Heranwachsenden in dieser Ordnung aufwirft. Wir greifen diese Frage am Schluss unseres Beitrages auf und stellen in aller Kürze eine Antwort vor, die eher eine Skizze als ein fertiges Gemälde ist und auf jeden Fall ausgearbeitet werden müsste.

Wir beginnen mit einer Begriffsklärung, die zu einem der wichtigsten Autoren führt, die sich zu diesem Thema geäußert haben: zu Immanuel Kant. An dessen Vorarbeiten knüpft auch Buck an.

\section{Der Begriff der Exemplarität}

Substantive mit dem Suffix -tät oder -ität, die seit dem Mittelhochdeutschen "hauptsächlich durch die Sprache der Kirche" und die höfische Literatur vermittelt worden sind, drücken eine Eigenschaft oder einen Zustand aus. ${ }^{14}$ Das Wort 'Exemplarität‘ bezeichnet demnach die Eigenschaft oder den Zustand des Exemplarisch-seins bzw., wie man genauer sagen müsste, es bezeichnet etwas, das der Sache nach exemplarisch ist für etwas anderes oder das ein Exemplar ist von etwas, das nicht es selbst schon von sich aus ist, oder das ein Exempel für etwas ist. Diese Genauigkeit ist nötig, weil es auch andere Substantive mit diesem Suffix gibt, die nicht in der gleichen Weise sowohl für etwas als auch von etwas relationiert werden. 'Subjektivität‘, 'Moralität‘ oder 'Fakultät‘ und 'Relationalität s sind Ausdrücke, bei denen der Doppelcharakter der Beziehung nicht sofort erkennbar ist und erst über Kontextualisierung shergestellt، wird. Bei 'Exemplarität ‘ wird das Beziehungsgefüge unmittelbar durch das Wort angezeigt, d.h. wer sexemplarisch،, 'Exemplar oder 'Exempel sagt, ist gezwungen, Auskunft darüber zu geben, für was etwas exemplarisch oder Exempel sein soll bzw. von was etwas ein Exemplar sein soll. Man benötigt zwingend eine Akkusativ- bzw. eine Dativ- oder Genitivergänzung, wenn man diese Wörter verwendet. Ansonsten sind solche Sätze in der deutschen Sprache bruchstückhaft.

Im modernen Wissenschaftsverständnis werden Eigenschaften und Zustände nicht mehr als Wesensmerkmale beschrieben, die den Sachen von sich aus zugehörig (ihnen seingeschrieben`) sind. Ebenfalls weggefallen ist die Beschreibung von Akzidentien, die einer Substanz anhaften, für diese aber nicht von ausschlaggebender Bedeutung (nicht sessentiells) sind. Was noch bleibt, ist eine Beschreibung der Welt mithilfe von Unterscheidungen, deren wiederholende Verwendung als Kategorien, Klassifikationsschemata, Ordnungsmuster, Denkformen oder Differenzfiguren 
selbst wiederum beschrieben werden können. Auf diese Weise sind umfangreiche Beschreibungen der Welt und Beschreibungen von Beschreibungen der Welt entstanden, die in der Wissenschaft als Formen der Theoriebildung zum Thema gemacht werden können. Im modernen Wissenschaftsverständnis stellt sich die Frage nach der Begründung und Absicherung von Aussagen daher mit besonderer Dringlichkeit.

Kennzeichnend für diese Art von Thematisierung ist, dass die Form der Relationierung an Bedeutung gewonnen hat. Beim Begriff der Exemplarität ist dies unvermeidlich, denn er rückt die Eigenschaft von etwas in den Blick, in Beziehung zu stehen. Etwas wird im Zustand des InBeziehung-Seins zum Thema gemacht. Entscheidend ist aus unserer Sicht, dass diese Relationierung nicht von einem festgeschriebenen Schema aus bestimmt werden muss. Es ist nicht schon alles fein säuberlich vorsortiert, wenn man anfängt, in der Pädagogik die Relationen einzusetzen, die Exemplarität zum Ausdruck bringen. Das wäre nur dann anzunehmen, wenn man die Ordnung des Erzieherischen doch wieder durch eine Wesensoder Substanzbeschreibung aufklären wollte.

Dass dies nicht erforderlich ist, zeigt bereits ein kurzer Blick auf den Begriff Exemplarität bzw. seine Unterscheidungen. $\mathrm{Da}$ Exemplarität heute kein vorrangiges Thema zu sein scheint, blicken wir in die Geschichte zurück bis zu der Zeit, in der Kant wirkte. Dort finden wir wichtige Begriffsklärungen, die auch heute noch nahezu unverändert im Spiel sind.

Im ,Grammatisch-kritischen Wörterbuch der hochdeutschen Mundart v von Johann Christoph Adelung finden sich die drei Einträge ,Das Exempelı, 'Das Exemplar` und 'Exemplarisch،. Der Eintrag ,Exemplarisch، lautet kurz und bündig: »im gemeinen Leben, andern zum
Exemplar, zum Muster dienend. Ein exemplarischer Wandel, ein strenger, tugendhafter Wandel, wenigstens dem Äußern nach. Ein exemplarischer Mann, der in seinem äußern Betragen andern zum Muster dienen kann. Jemanden exemplarisch strafen, so dass andere ein Exempel daran nehmen können, strenge. "Das 'Exempel stammt vom Lateinischen "Exemplum, das Beyspiel, in beyden Bedeutungen dieses Wortes «. Adelung erläutert: »1) So fern es eine ähnliche Sache ist, welche die Möglichkeit einer andern zeiget, oder ihr zur Erläuterung dienet. Ein Exempel anführen. Etwas zum Exempel anführen. [...] 2) In engerer Bedeutung, eine Begebenheit, die man zur Vorschrift seines Verhaltens annimmt, oder annehmen soll; ein Beyspiel, Vorbild. Ich will deinem Exempel folgen. Laß dir das ein Exempel seyn. Ein Exempel an etwas nehmen, sich eine Begebenheit zur Warnung dienen lassen." Das 'Exemplar wird als "ein Muster, ein Vorbild seines Verhaltens, wie Exempel« eingeführt. Jemand ist ein "Exemplar der alten Redlichkeit«. Allerdings, sagt Adelung, komme das Wort in dieser Bedeutung "wenig mehr vor «. Es gehört in dieser Bedeutung in den semantischen Hof von exemplarisch. In einer anderen Bedeutung wird ,Exemplar terminologisch verwendet. "2) Bey den Buchdruckern bedeutet Exemplar das Original einer Schrift, dasjenige, was bey dem Setzen eines Buches oder einer Schrift dem Setzer zum Muster dienet. 3) Bei den Buchhändlern hingegen ist Exemplar ein Stück der ganzen Auflage, ein Buch oder eine Schrift als ein Individuum betrachtet. Ein Exemplar von Gellers Moral. Sechs Exemplare der Deutschen Bibel. «" 15

In Georg Samuel Albert Mellins ,Encyklopädischem Wörterbuch der Kritischen Philosophie، finden sich auf zwei Seiten Hinweise auf Kants Ausführungen zum 'Exemplarischen،. An folgende Hinweise wäre aus unserer Sicht zu erinnern: 
"Exemplarisch heißt«, schreibt Mellin, "alles, was nicht nachgeahmt ist, und doch nachgeahmt werden muss «. Exemplarisch "heißt etwas, wenn es Muster seyn kann. Die Werke oder Producte des Genies z.B. sind exemplarisch, weil sie die Muster seyn müssten, nach welchen ähnliche Werke gearbeitet und beurtheilt werden müssen. [...] Das Exemplarische ist dem Nachgeahmten entgegengesetzt." Das Produkt eines Genies erkennt man daran, dass es "nicht durch Nachahmung entsprungen" ist. Es kann ein Produkt nachgeahmt sein und gleichwohl Exemplarisches enthalten, d.h. etwas zur Darstellung bringen, was "zum Muster dienen kann«. Ebenso kann das Produkt eines Genies etwas enthalten, "worin es nicht exemplarisch ist". In diesen Passagen hat das "Talent" des Genies "geschlummert «. ${ }^{16}$

In Kants 'Kritik der Urteilskraft، stößt man auf wichtige Ergänzungen, die von Mellin nicht erwähnt werden. Dass Exemplarische ist nicht identisch mit Originalität, weil "es auch originalen Unsinn geben kann«, den niemand sich zum Vorbild nehmen sollte, heißt es bei Kant. Zum Vorbild nehmen meint, dass etwas "zum RichtmaBe oder Regel der Beurtheilung « gewählt wird. Wie man zu dieser Regel kommt und warum man sich ihr unterwirft, kann das Genie "selbst nicht beschreiben, oder wissenschaftlich anzeigen «. Es kann nur als der "Urheber « einer Sache bezeichnet werden, der "selbst nicht weiß, wie sich die Ideen dazu herbei finden, auch es nicht in seiner Gewalt hat, dergleichen nach Belieben oder planmäßig auszudenken und anderen in solchen Vorschriften mitzutheilen, die sie in Stand setzen, gleichmäßige Produkte hervorzubringen «. ${ }^{17}$

Um etwas als geeignetes Muster für eigenes Verhalten identifizieren zu können, muss man schon selbst "Geschmack" haben. Wer Geschmack hat, ist darum aber noch nicht selbst ein Genie, das Exemplarisches hervorbringen kann. ${ }^{18} \mathrm{Er}$ verfügt vielleicht über "Geschicklichkeit“ in der Nachahmung des Musters. Diese ist zu unterscheiden von der Fähigkeit des Geschmacks, das Muster als geeignet für die Orientierung eigenen Verhaltens zu beurteilen. Das Maß, an dem ein Geschmacksurteil gemessen werden muss, kann niemals eine empirische Erfahrung sein (es kann nicht durch Nachmachen erworben werden), sondern es muss als Idee angelegt sein. Diese muss "jeder in sich selbst hervorbringen «, um sie an ein Verhalten anlegen zu können, das als Exemplar gedeutet wird. ${ }^{19}$ Ein Exemplar ist nicht von sich aus Exemplar, sondern durch das Urteil, das jemand fällt, indem er die Idee des guten Geschmacks für sich entwickelt und als Kriterium der Beurteilung von Verhalten aller Art anwendet.

Halten wir fest: Am Begriff der Exemplarität wird unterschieden die Originalität eines Musters bzw. Vorbildes. Diese Relation bezeichnet der Ausdruck 'Exempel،. Am Begriff wird weiterhin unterschieden das Beispielhafte eines Verhaltens. Dieses wird durch den Ausdruck sexemplarisch، markiert. Schließlich unterscheidet man am Begriff der Exemplarität noch den Exemplar-Status, den etwas relativ zu einem Zusammenhang hat, dem es zugeordnet ist. Um diese Differenzen erfassen und beurteilen zu können, ist Geschmack nötig, den jemand von sich aus aufbringen und an Gegebenheiten anlegen muss. Der Maßstab der Beurteilung ist nicht durch Nachahmung zu finden, weil er keine empirische Erfahrung ist, sondern Einsicht in eine Idee voraussetzt.

\section{3. »Moralischer Monismus«}

An diese Unterscheidungen knüpft Günther Buck an. Er vertieft und erweitert sie, indem er sich mit Kants ,Lehre vom Exempel auseinandersetzt und diese um pädagogische Fragestellungen ergänzt. ${ }^{20}$ 
Buck setzt an bei der Frage, wie Allgemeines und Besonderes in ihrer wechselseitigen Bedingtheit bestimmt werden können. Kant hatte bekanntlich einen Vorschlag gemacht, der die Allgemeinheit der Maxime, die den einzelnen bindet, der Singularität von je spezifischen Entscheidungssituationen gegenüberstellt. Orientierung finden sollte der Handelnde an einer Maxime, deren Allgemeinheit den Wandel der Ereignisse und Herausforderungen übergreift. Diese Auffassung mündet in die berühmte Formel des $\mathrm{Ka}$ tegorischen Imperativs ein: "Handle so, dass die Maxime deines Wollens jederzeit Prinzip einer allgemeinen Gesetzgebung sein könnte. « ${ }^{21}$

Die Gegenposition zu diesem Vorschlag hebt die Individualität des Handelnden und die je spezifischen singulären Situationsbedingungen hervor, unter denen Entscheidungen für Handlungen getroffen werden. Orientierung kann es nach diesem Verständnis nur in einer Anpassung an die Veränderbarkeit geben, wozu den jeweiligen singulären Ereignissen und Bedingungen Rechnung getragen werden muss. In zugespitzter Form hat Nicolai Hartmann diese Kritik gegen Kants kategorischen Imperativ gewendet: "Handle so, daß die Maxime deines Willens niemals zugleich (wenigstens niemals restlos zugleich) Prinzip einer allgemeinen Gesetzgebung sein könnte. «22

Hartmann zog damit die Konsequenz aus der Kritik, die bei Friedrich Nietzsche vorbereitet, von diesem aber ins Extrem des individuellen Relativismus getrieben worden war. Hartmann wollte das Extrem vermeiden und gleichwohl der Individualität und Singularität des moralischen Urteilens und Handelns Rechnung tragen. Er griff dazu Kants Formulierung des kategorischen Imperativs an einem Punkt an, den er als "moralischen 'Monismus « bezeichnete. Dessen Fehler ist, "dass das Gute einseitig beschränkt wird, dass von einem begrenzten Wert behauptet wird, er sei das ganze Gute «. ${ }^{23}$

Hartmann monierte, das Allgemeine, an dem das Besondere des moralischen Urteils Maß nehmen soll, würde in der Geschichte der Moralphilosophie immer auf die am weitesten abstrahierbare Ebene der Glückseligkeits-, Nützlichkeits-, Gerechtigkeits-, der Liebes-, der Kraft- oder der Herrenmoral gehoben, auf der alle Differenzen nivelliert sind und es streng genommen nichts mehr zu urteilen gibt, weil es nur noch darum geht, das jeweils aktuelle Ereignis einem vorgängig aufgestellten Schema einzuordnen. Die monistische Auffassung von Moralität hat die Aufgabe des moralischen Urteilens degradiert zur Sammlung von Bestätigungen des Guten, das schon feststeht. Von moralischem Urteilen kann man dann nicht mehr sinnvoll sprechen, wenn man nur noch abhakt, was im Lichte der vorgegebenen Auffassung als moralisch gut und böse zu gelten hat. Mit der Starrheit des Schemas ist das Lebenselixier des Urteilens überhaupt vernichtet: die Freiheit der Wahl, die ohne die Qual der Begründung nicht zu haben ist.

\section{4. "Methodenlehre der moralischen Bildung “}

Gegenüber dieser Kritik versucht Buck Kants Position stark zu machen, indem er die pädagogischen Stellen in dessen Werk als "eine Methodenlehre der moralischen Bildung " auslegt. "Das ausgezeichnete Mittel aber der moralischen Bildung, d.h. der Gründung eines Charakters ist das Beispiel."Am Beispiel, so Buck, können wir lernen, was es heißt, moralisch zu sein, ohne unser Verhalten in ein vorgegebenes Schema pressen zu müssen. Um einem weit verbreiteten Missverständnis vorzubeugen, lehnt Buck gleich zu Anfang die "banale Illustration-Theorie " ab, die den Zweck eines Beispiels darauf reduziert, 
etwas Allgemeines anschaulich darzustellen. ${ }^{24}$ Das mag ein Motiv sein, das eine gewisse Rolle in pädagogischen Zusammenhängen spielt. Es ist aber keineswegs das einzige und schon gar nicht das entscheidende Motiv.

Buck denkt die Relation Allgemeines | Besonders, die am Beispiel exemplifiziert wird, grundlegender. Er geht davon aus, dass das Allgemeine die Wahl des Beispiels bestimmt und ordnet dem Beispiel die Funktion zu, das Allgemeine angemessen darzustellen. Angemessenheit muss aber von Anschaulichkeit unterschieden werden. Ein Beispiel erweist seine Eignung zur Darstellung eines Allgemeinen folglich, wenn es angemessen ist; ob es auch anschaulich ist, ist hingegen zweitrangig. Dies hat Konsequenzen: Wenn ein ungeeignetes Beispiel gewählt wird, dann weil es nicht zum Allgemeinen passt. Wenn ein ungeeignetes Beispiel gewählt wird, dann wird das Allgemeine verfehlt, das durch jenes dargestellt werden sollte, mag das Beispiel auch noch so anschaulich sein. Seine Anschaulichkeit ist kein Vorteil, wenn das Beispiel nicht zum Allgemeinen passt. ${ }^{25}$

In der Erziehung, so Buck, wählt eine Person ein spezifisches Beispiel deshalb nicht aus dem Grund, dass inm die Worte fehlen, um einen allgemeinen Sachverhalt einem anderen anschaulich, d.h. Punkt für Punkt nachvollziehbar, darzulegen. Sie greift nicht zu dem Mittel der bildhaften Vorstellung, weil sie meint, in der simultan gegebenen Einheit des Bildes das ausdrücken zu können, was ihr in der sequentiellen Ordnung des Sprechens nicht gelingen will. Buck hebt vielmehr hervor, dass das Sprechen selbst bereits ein Beispiel ist, das in der Kommunikation wahrgenommen werden kann. Es ist in dieser Funktion "das gute Beispiel an dem Lehrer selbst (von exemplarischer Führung zu sein) «, wie Kant sagt. ${ }^{26}$ In diesem Fall ist es das Exempel, von dem in den eingangs erwähnten Lexikonartikeln gesagt wurde, es stehe für eine Sache, "welche die Möglichkeit einer andern zeiget, oder ihr zur Erläuterung dienet«. Das Exempel bringt einen Sachverhalt zum Ausdruck, d.h. es zeigt an, wie es sich mit der Sache verhält, indem über sie gesprochen wird.

Wer über eine Sache spricht, sagt Buck, der zeigt mit seinem Verhalten, welche Möglichkeit des Sprechens-über ergriffen und wie das Sprechen-über in den sumgreifenden Kontext menschlichen Miteinanderumgehens eingeordnet werden kann. Das Sprechen erläutert sich quasi selbst, indem es sich der Wahrnehmung präsentiert und der Auslegung zur Verfügung stellt. Es präsentiert, indem es in der Anwesenheit eines anderen vollzogen wird, die Regeln, die ein Sprechen dieser Art erfüllen muss, um spezifischen Erwartungen zu entsprechen, ohne dass diese Regeln selbst explizit, also in Satzform ausgesprochen werden müssen. Sie müssen aber gedeutet werden, um das Verhalten als Fall-von Regelanwendung identifizieren zu können. Für sich betrachtet ist Verhalten nicht mehr als körperliche Bewegung im Raum, die an einem Organismus wahrgenommen wird. So betrachten z.B. Physiker die Welt. Für sie gibt es keinen Grund, Exemplarisches zu entdecken, sie kommen zurecht, wenn sie in Beispielen sprechen, um Fälle von empirisch nachgewiesenen Gesetzen anschaulich zu beschreiben. In der Einstellung des Naturwissenschaftlers wird das Desinteresse an der Exemplarität von Handlungen zur methodischen Norm erklärt, die eingehalten werden sollte, um dem Ideal der Naturwissenschaften möglichst nahe zu kommen: der Beschreibung einer Welt, wie sie beschaffen sein müsste, wenn man alle subjektiven Beschreibungen aus ihr herausrechnet, d.h. eine Welt, wie sie wäre, wenn sie nicht beschrieben würde.

Buck weiß, dass dies nicht der Zweck einer pädagogischen Beschreibung sein 
kann. Das pädagogische Sprechen-über bringt nämlich Sachverhalte zum Ausdruck, die in sich kommunikativ reflektiert sind. Es stößt deshalb auf das Problem, wie das Zusammenspiel gelingen kann, das sich in diesen kommunikativen Reflexionen entfaltet.

\section{5. "Thunlichkeit oder Unthunlichkeit einer Handlung «}

Buck übernimmt hier die von Kant vorgeschlagene strikte Unterscheidung der Worte Beispiel und Exempel: "Woran ein Exempel nehmen und zur Verständlichkeit eines Ausdrucks ein Beispiel anführen, sind ganz verschiedene Begriffe."Während das Beispiel, wie Kant in der >Metaphysik der Sitten، sagt, "blos die theoretische Darstellung eines Begriffs ist«, d.h. nicht mehr als etwas Allgemeines in besonderer Darbietung präsentiert, ist das Exempel "ein besonderer Fall von einer praktischen Regel, sofern diese die Thunlichkeit oder Unthunlichkeit einer Handlung vorstellt «. ${ }^{27}$ Von Kant ist diese Unterscheidung durchaus als eine terminologische Differenz, d.h. als eine theoretisch geführte Beobachtungs- und Sprachordnung, verstanden worden. Wenn wir uns an sie halten, ergibt sich folgende Konsequenz: Die handelnde Person kann sich nicht selbst zum Exempel für die "Thunlichkeit oder Unthunlichkeit einer Handlung " erklären, weil sie ihr Handeln lediglich in Beispielen verständlich machen kann. Auch wenn sie noch so sehr davon überzeugt sein sollte, ,thunlich، oder sunthunlich، zu handeln, muss sie akzeptieren, dass erst in der Kommunikation beurteilt werden kann, ob ihr Verhalten als ein Fall von der Regel gelten kann, deren Befolgung ihre Handlung als sthunlich und sunthunlich überhaupt erst identifizierbar macht. Im Umgang miteinander wird das Verhalten einer Person erst und nur dadurch zum Exempel, dass es als Ausdruck eines Regelverständnisses im Hinblick auf eine Handlung gedeutet wird.

Buck weist an dieser Stelle ausdrücklich darauf hin, dass diese Deutung von Beispiel und Exempel bei Kant auf Anwesenheitsbedingungen bezogen ist. Beide Begriffe sind bei ihm nur sinnvoll unter der Bedingung, dass jemand die Erfahrung macht, mit einem anderen gemeinsam zu kommunizieren und der andere dabei leibhaftig anwesend ist. ${ }^{28}$ Kant hat die Unterstützung der moralischen Bildung in erster Linie als Nahraum-Erfahrung thematisiert, obwohl er um die moralische Wirkung von schriftlichen Texten wusste. Eine pädagogische Situation war für inn allerdings noch nicht unter den Bedingungen von face-to-interface-Konstellationen denkbar, die wir heute kennen, wenn wir mit jemandem auf digitalem Wege kommunizieren.

Unter den Anwesenheitsbedingungen einer Kommunikation wird die Regel, die jemand in seinem Verhalten zum Ausdruck bringt, in Bezug auf die "Thunlichkeit oder Unthunlichkeit einer Handlung “ gedeutet, und zwar von einer anderen Person, die das Verhalten wahrnimmt. Sie ist frei darin, das Verhalten der von ihr wahrgenommenen Person gemäß ihren eigenen Erwartungskriterien als Exempel guten Handelns zu deuten. Es ist dafür nicht nötig, dass die wahrgenommene Person ihr eigenes Verhalten als Beispiel des guten Handelns explizit kommuniziert. Denkbar ist auch, dass die Person gar nicht bemerkt, dass sie unter moralischen Gesichtspunkten beobachtet wird. Im Grunde ist dies sogar der Idealfall, denn »Moralität kann nicht nur nicht belegt werden, sie soll auch gar nicht belegt werden «, sagt Buck. ${ }^{29}$ Moralität kann durch Wahrnehmung des Verhaltens einer Person erkannt werden, indem eine andere Person dieses Verhalten unter dem Gesichtspunkt der "Thunlichkeit oder Unthunlichkeit“ beurteilt und eine Regel in ihm entdeckt, deren Befolgung es als gutes oder schlechtes Handeln ausweist. 
Buck ist hier eindeutig: Moralität kann - als Moralität - nicht doziert werden. Sie kann nur erkannt werden an einer Person, die von sich aus die "Thunlichkeit" einer Handlung zum Ausdruck bringt, wenn und indem eine andere Person in der Lage ist, einen Maßstab anzulegen, der es erlaubt, das Verhalten der Person unter dem Gesichtspunkt der Moralität zu beurteilen. Kurzum: Beide Personen - die handelnde, wie die sie beobachtende - müssen sich relativ zur Moralität bestimmen können. Sie müssen, wie Kant sagt, das Sittengesetz schon voraussetzen, um ein spezifisches Verhalten als Fall diesem Gesetz subsumieren zu können. Sie müssen aber auch die Gelegenheit haben, das Verhalten wechselseitig zu beurteilen. Das Miteinanderumgehen muss so gestaltet sein, dass jede Person sich als Original der Beurteilung von Handlungen erfahren kann.

Jede Person beurteilt das Verhalten der von ihr wahrgenommenen Person relativ zum Sittengesetz, von dem angenommen wird, dass es für alle anwesenden Personen gleichermaßen verbindlich ist und als vernünttig eingesehen werden kann. In diesem Sinne tritt jede Person als ein Original der Auslegung des Sittengesetzes im Miteinanderumgehen in Erscheinung. Man kann sich wechselseitig als ein Exempel dafür deuten, wie das Sittengesetz ausgelegt werden kann und sollte. Menschen identifizieren sich sozusagen wechselseitig als Beispiele für Moralität, die möglich ist, wenn sie das Miteinanderumgehen im Lichte des Sittengesetzes betrachten.

\section{Sittengesetz}

Buck gibt an dieser Stelle einen wichtigen Hinweis: Das Sittengesetz wird nicht in der Form vorausgesetzt, dass es z.B. in einer schriftlich kodifizierten Form nachlesbar vorliegt. Es gibt nicht das Buch 'Sittengesetz‘, das im Gericht liegt und anhand von Artikeln und Paragraphen regelt, was wann zu tun ist. Deshalb kann das Handeln der Person nicht darin bestehen, die Artikel und Paragraphen vorzulesen und der beobachtenden Person abzuverlangen, das Vorgelesene zu lernen, um es an anderer Stelle so zu repetieren, dass es unter Rückgriff auf die Artikel und Paragraphen als zutreffend oder nichtzutreffend beurteilt werden kann.

Die Voraussetzung des Sittengesetzes ist vielmehr so zu verstehen: Die handelnde Person führt eine Handlung an etwas aus und sie zeigt in der Ausführung dieser Handlung, dass sie sich zu einem Sittengesetz verhält, das nirgends geschrieben steht und auf das man sich nicht im empirischen Verständnis berufen kann. Die sie beobachtende Person deutet die Handlung als einen angemessenen Ausdruck der Orientierung am Sittengesetz, wenn und weil sie zu diesem Verhalten "Zutrauen « fassen kann. Sie stimmt in dieses Verhalten ein, weil sie von seiner Angemessenheit überzeugt ist. "Dieses wohlbegründete Zutrauen zur Gesinnung des anderen, diese Antizipation seines guten Willens, die konstitutiv für das Exempel und seine rechte Auffassung ist, ist nun der Grund dafür, dass derjenige, der sich vom Exempel etwas sagen lässt, Zutrauen zu sich selbst und seinem Vermögen fasst, dem Sittengesetz zu gehorchen. Die 'Ermunterung d durch das Exempel gründet im Zutrauen zur Gesinnung des anderen, dessen Verhalten nun zum Exempel wird. ${ }^{30}$ Die Berufung auf die beurteilte "Thunlichkeit oder Unthunlichkeit“ des Handelns unter seinesgleichen ist somit der Nachweis des Sittengesetzes.

Die Bildung eines moralischen Charakters gelingt in dieser Lesart dadurch, dass das Verhalten einer Person als "Urbild (exemplar) « für die "Thunlichkeit einer Handlung « erkannt wird, weil die sie beobachtende Person "Zutrauen« fassen kann. Für die beobachtende Person hat das in 
ihrer Anwesenheit gezeigte Verhalten einladenden Charakter. Wer die Einladung annimmt, wer "Zutrauen« in dem geschilderten Sinne fasst, kann selbst zum Exemplar der Beurteilung moralischen Handelns werden.

Buck betont, dass das Exemplar nicht in Relation zu einem wahrnehmbaren, vorgegebenen Bestand, dem niedergeschriebenen Sittengesetz, erkannt wird. "Dasjenige, worauf das Exemplarische verweist, ist sunbestimmt, es hat Dynamis-Charakter, d. h. es wird durch jede neue Konkretion weiterbestimmt. «31 Die Dynamik kommt durch den Einsatz der Urteilskraft ins Spiel, die in immer neuen Anläufen geübt wird. Geübt wird nicht die Regel, die jemand doziert, sondern ob das Verhalten ein Beispiel für Regelbefolgung ist. "Die das Beurteilungsvermögen (zu entscheiden, ob etwas der Fall einer Regel ist) übenden Beispiele sind ja keineswegs Beispiele für die Regel, sondern Beispiele für die Beurteilung vorliegender - strittiger - Fälle nach der Regel." Denn: "Es gibt keine angebbare Regel für die Beurteilung nach Regeln. ${ }^{32}$

\section{7. "Gesetz der Bipersonalität oder Zweisamkeit“}

Die bisherige Überlegung hat zu folgendem Ergebnis geführt: Exemplarität kommt ins Spiel, ohne dass ein Zusammenhang erkennbar wäre, der die Unterscheidungen nach eindeutig angebbaren und allgemein verbindlichen Regeln ordnet. Die Personen machen das Phänomen der Exemplarität unter sich aus, ohne dass sie dazu auf eine vorgegebene Ordnung zurückgreifen könnten. Sie agieren, wie Markus Gabriel sagt, nicht in "einem einzigen Gesamtzusammenhang, der alles Heterogene uniformiert, eine Welt", weil es keine einheitliche Reflexionsperspektive auf die Welt gibt. ${ }^{33}$ Die Perspektiven können nur im Plural identifiziert werden, so dass man den Begriff ,Welt ‘ nur noch zur Kennzeichnung der Perspektivität von Betrachtungen verwenden kann.

Das gilt uneingeschränkt auch für die Erziehung, denn Erziehung ist eine der Formen des Miteinanderumgehens, die dem "Gesetz der Bipersonalität oder Zweisamkeit“ unterliegen, wie es Viktor von Weizsäcker formuliert hat. "Darunter verstehe ich«, schreibt er, "die irreduzible Zweiseitigkeit der sittlichen Wirkung, derzufolge sie nur als Verhältnis zweier Personen überhaupt der Wirklichkeit angehören kann, nur als solches Verhältnis vollziehbar, aber auch nur als solches rational denkbar, vorstellbar ist. « ${ }^{34}$ Die Irreduzibilität der Form kommt in der Erziehung dadurch zum Ausdruck, dass alles, was sie ausmacht, durch ein Miteinanderumgehen von Personen realisiert werden muss. Mit anderen Worten: Alles ist relativ zum Miteinanderumgehen bestimmt, wenn man Erziehung pädagogisch in den Blick nimmt.

Wendet man diese Annahme konsequent an, dann führt sie zur Einsicht in die Selbstreferentialität der Form des Miteinanderumgehens, denn auch die Personen können nur relativ zu der Form des Miteinanderumgehens bestimmt werden, die sie hervorbringen. Der Begriff ,Form des Miteinanderumgehens، bzw. der "Bipersonalität oder Zweisamkeit « ist der Komplementärbegriff zu ’Person‘. Mit anderen Worten: Vor dem Miteinanderumgehen oder außerhalb der "Bipersonalität « gibt es die Personen nicht, die erst im Miteinanderumgehen identifizierbar werden. Da im pädagogischen Denken die Genese der Person eine zentrale Rolle spielt, können wir auch sagen: Gleichzeitig mit der spezifischen Form des Miteinanderumgehens entsteht die Person, wie umgekehrt mit der Bildung der Person die Form des Miteinanderumgehens Gestalt gewinnt. Für die weiteren Ausführungen ist es wichtig festzuhalten, dass dies für beide Personen 
gilt, die in der irreduziblen "Bipersonalität « bestimmt werden: für den Erzieher und den Edukanden.

Man erkennt die Irreduzibilität der Form, die wir Erziehung nennen, bereits daran, dass die Begriffe, mit denen wir die Personen bezeichnen, nur als Relationsbegriffe Sinn ergeben. Auf einen Erzieher ohne Zögling bzw. Edukand kann man sich keinen Reim machen. Das gilt umgekehrt genauso: Von einem Zögling bzw. Edukanden ohne eine Person, die als Erzieher in Erscheinung tritt, zu reden, ergibt keinen Sinn. Erziehung ist als Form deshalb nur dann 'geschlossen`, wenn die wechselseitige Bezugnahme von Erzieher und Edukand gegeben ist. Wo die Geschlossenheit der Form aufgebrochen wird, wird das Verhältnis von Personen nicht als ein pädagogisches thematisiert. Man redet dann über Personen, ohne sie relativ zu einer Form des Miteinanderumgehens zu betrachten, die durch ihre Aktivitäten entsteht, stabilisiert und verändert wird. ${ }^{35}$

Wo es zu einer Reduktion kommt, wird die Dynamik eingefroren, die für das Miteinanderumgehen von Personen kennzeichnend ist. Mit Hannah Arendt gesprochen, wird in einer solchen Beziehung nicht mehr gedacht, wenn Denken als die Aktivität verstanden wird, durch die die Fähigkeit zum Ausdruck kommt, "vom Gesichtspunkt eines anderen Menschen aus zu denken «. ${ }^{36}$ Wenn der andere zum reinen Objekt reduziert wird, ist es weder nötig noch möglich, von seinem Gesichtspunkt aus zu denken. Mit ihm kann als $\mathrm{Ob}$ jekt gerechnet werden.

Für das Thema 'Exemplarität‘ wäre es insofern von Interesse, der Frage nachzugehen, wie eine Ordnung des Erzieherischen, die durch die Aktivitäten der Personen als eine konkrete Form des Miteinanderumgehens entsteht, stabilisiert und verändert wird, möglich ist. Eine Antwort auf diese Frage müsste auch Auskunft darüber geben, wie eine solche
Ordnung gesichert werden kann, um der heranwachsenden Person das "Zutrauen" in Exempel für die "Thunlichkeit der Handlung « zu ermöglichen, damit sie die Erfahrung machen kann, selbst ein Original der Beurteilung der »Thunlichkeit und Unthunlichkeit« des Handelns unter seinesgleichen zu sein, ohne dass man sie auf ein vorgegebenes Sittengesetz abrichtet. Mit anderen Worten: Der heranwachsende Mensch soll die Erfahrung machen können, ein Exemplar der Menschheit zu sein, das als Exempel für gelingendes Leben taugen könnte - nicht im Sinne eines künstlerischen Genies, wohl aber als Partner des Miteinanderumgehens.

\section{Von der Form des Miteinanderumgehens zum Rechtstatus in der Erziehung}

Bucks Überlegungen setzen voraus, dass die naive Vorstellung von Pädagogik als Technik der Erziehung aufgegeben wird. Erziehung ist nicht einfach Einwirkung eines Menschen auf einen anderen, sondern ein intersubjektiver Handlungszusammenhang, der einen unauflösbaren Rest von Unbestimmtheit enthält, der mit Urteilskraft und Einfühlungsfähigkeit zu begegnen ist. ${ }^{37}$ Damit das "Zutrauen « als Bedingung moralischer Bildung, von der Buck gesprochen hatte, möglich sein kann, sind Vorstellungen von gelingendem Leben und Zusammenleben gemeinsam auszuhandeln. Dieses "Zutrauen « ist unerlässlich, wenn sich ein Kind als das Original des Urteils über die "Thunlichkeit und Unthunlichkeit der Handlung « des Erziehers empfinden soll.

Der erziehende und der zu erziehende Mensch tragen mit ihren Aktivitäten wechselseitig dazu bei, dass Erziehung gelingt oder scheitert. Die Mensch- bzw. Personwerdung ist somit eng mit dem Stellenwert des sich entwickelnden Individuums 
im konkreten Miteinanderumgehen verbunden und es stellt sich die Frage, ob es eines eigenen Rechtsstatus bedarf, damit der heranwachsende Mensch sein Verständnis von "Exemplar-Sein « zu entwickeln und in die Kommunikation einzubringen vermag. Diese Frage erscheint uns als berechtigt und wichtig, weil der Stellenwert im sozialen Miteinander nicht schon allein dadurch bestimmt wird, dass ein Individuum sich die Freiheit nimmt, Position zu beziehen, und auch nicht schon allein dadurch, dass inm von anderen ein Platz im sozialen Geschehen zugewiesen wird. Der Status als Rechtssubjekt verknüpft beide Dimensionen, da er das Verhältnis von Rechten und Pflichten zur Aufgabe erklärt und die interagierenden Individuen zur Beurteilung von Grenzen der Selbstund Fremdbestimmung auffordert.

Die Erfüllung dieser Aufgabe setzt die Herausbildung von Fähigkeiten voraus, die nicht in privater Isolation erworben werden können. In modernen Gesellschaften sind Fähigkeiten dieser Art Gegenstand von pädagogischem Handeln. Es muss daher bekannt sein, was es zu unterstützen gilt, damit die erzieherische Hilfe angemessen gestaltet werden kann. Mit Buck gesprochen, geht es neben vielem anderen auch darum, dem heranwachsenden Menschen die Gelegenheit einzuräumen, sich als eine Person erfahren zu können, denn als 'Personen bezeichnen wir die Lebewesen, mit denen wir als unseresgleichen kommunizieren und mit denen wir anders umgehen als mit Dingen, Maschinen oder Tieren. Nur Personen können wir als Partner ansprechen, die mit uns Beurteilungen von Handlungen unter einem Sittengesetz der Menschheit vornehmen. Nur im Umgang mit Personen können wir hoffen, in dieser Aufgabe weiterzukommen. Personen sind nämlich, mit Kant gesprochen, Lebewesen, die wir als vernunftbegabt ansehen und denen daher Würde zukommt. Ihnen bringen wir, wie er sagt, Achtung entgegen, indem wir sie nicht nur als Mittel eigener Zwecksetzung, d.h. nur als Objekte unseres Denkens behandeln, sondern indem wir ihnen den Status zusprechen, selbst Zwecke zu setzen. Sie sind, was kein Stein, keine Maschine und kein Tier bisher zu sein beansprucht hat: Subjekte einer gemeinsamen Geschichte. Mit Kant, Jaspers, Arendt und Buck kommt aus diesem Grunde Humanität ins Miteinanderumgehen, wenn vom Gesichtspunkt eines anderen Menschen aus gedacht wird. Diese Geste bringt erst eine Haltung zum Ausdruck, die den Status eines mit Würde begabten Lebewesens anerkennt, "für welchen das Wort Achtung allein den geziemenden Ausdruck der Schätzung abgiebt, die ein vernünftiges Wesen über sie anzustellen hat «. ${ }^{38}$

Die Ausbildung einer Identität sowie die Entwicklung der nötigen Selbstachtung und Achtung gegenüber anderen Menschen stellt vor diesem Hintergrund eine zentrale Entwicklungsaufgabe eines Menschen dar, die offenbar nur gelingen kann, wenn das Kind eine entsprechende Achtung erfährt und lernt, diesen Status für sich zu beanspruchen. ${ }^{39}$ Wenn sich das Interesse auf das Einmalige, das Unwiederholbare, das Original richten soll - alles Eigenschaften, die Menschen und damit Kindern, insofern sie unter dem Kriterium der Exemplarität betrachtet werden, zugeschrieben werden -, wie ist dann der Status eines Kindes zu denken, so dass es nicht einfach nur in eine vorgegebene Erziehung hineingestellt und damit Erwachsenen gegenübergestellt und von ihnen auf den Status eines Objektes reduziert wird?

Erziehungswissenschaftliche Auseinandersetzungen zum Rechtsstatus von Kindern in der Erziehung existieren bislang kaum, ebenso wenig scheint es eine pädagogische Theorie zu geben, in der geklärt ist, dass Kinder Rechtssubjekte sind. Dabei gibt die Existenz eines 
Menschenrechtsabkommens ausschließlich für Kinder und Jugendliche - die 1989 in Kraft getretene UN-Konvention über die Rechte des Kindes - Anlass, über dieses nicht nur pädagogische, sondern interdisziplinäre Kernproblem nachzudenken. Nicht zuletzt offenbart die Auseinandersetzung mit dem Rechtsstatus von Kindern auch die Ansichten einer Gesellschaft über Kinder und was es bedeutet, Kind oder Nicht-Kind zu sein.

Die Besonderheit und Herausforderung ist deutlich erkennbar: Zunächst nehmen andere Menschen, d.h. Eltern, Betreuungspersonen und der Staat, die Rechte von Kindern und Jugendlichen ${ }^{40}$ wahr und interpretieren sie inhaltlich, ehe diese Kompetenzen nach und nach auf die sich entwickelnden Kindern und Jugendlichen übergehen können. Geprägt sind diese Auseinandersetzungen über die Bedingungen der Möglichkeit von Rechtsstatus von einem der Thematik immanenten Spannungsfeld, indem einerseits die Schutzbedürftigkeit von Kindern betont und eingefordert wird, andererseits Kinderrechte als Ausdruck und Mittel von und zur Emanzipation postuliert werden. ${ }^{41}$

Um den Rechtstatus des Kindes von einer pädagogischen Warte aus zu betrachten, bietet sich die "Bildsamkeit des Zöglings" als "Grundbegriff der Pädagogik « ${ }^{42}$ in der Tradition Johann Friedrich Herbarts an, der sich um eine Theorie bemühte, die das Miteinanderumgehen von Erzieher und Zögling bzw. Edukand thematisiert. Bildsamkeit bezeichnet die anthropologische Tatsache, dass der Mensch als bedürftiges, imperfektes und in Entwicklung begriffenes Wesen auf die Welt kommt. Deshalb wird er in der Pädagogik angesehen als ein Lebewesen, das auf Erziehung angewiesen, erziehungsbedürftig und fähig ist, diese Unterstützung anzunehmen. Er ist, so die Generalprämisse, auf Erziehung ansprechbar und lernfähig. Ab dem Moment seiner Geburt steht der Mensch in einer Geschichte der Erziehung, in der er sich in der Auseinandersetzung mit seinem Erzieher beziehungsweise seiner Erzieherin, auf jeder Altersstufe spezifische "Fähigkeiten des Weiterkommens" aneignet, die das Ergebnis vergangenen und die Ausgangslage zukünftigen Miteinanderumgehens von Erzieher und Edukand sind. ${ }^{43}$ Mit anderen Worten: Der erziehende und der zu erziehende Mensch stehen in Beziehung zueinander und haben vergangene sowie zukünftige Erziehungserfahrungen. Bildsamkeit setzt damit die Möglichkeit voraus, die Entwicklung eines Menschen durch erzieherische Tätigkeiten zu fördern und ist eine "jedem Menschen zuzuerkennende, 'zunächst d durch keinerlei individuelle und biographische oder soziale und historische Gegebenheiten eingegrenzte Voraussetzung « ${ }^{44}$ Das Vorhandensein von Bildsamkeit - und damit einhergehend die Möglichkeit pädagogischen Einflusses kann nicht sinnvoll bestritten werden und ist vorauszusetzen, damit Erziehung und Beschreibungen von Erziehung sinnvoll sein können.

Der "Grundbegriff der Pädagogik“ bezeichnet also einerseits den Umstand, dass Kinder und Jugendliche als bildsame Lebewesen angesehen werden sollen, und stellt andererseits eine Beschreibungskategorie zur Verfügung, welche hilft, die komplexe Welt von Erziehung und Bildung zu ordnen. Unabhängig davon, aus welcher Perspektive Erziehung ansetzt, nach Herbart muss sie sich letztendlich an der Bildsamkeit des Menschen ausrichten, um ihm zu helfen, "das zu werden, was er einmal wünschen wird, geworden zu seyn «. ${ }^{45}$ Auch in der Moderne wird die Aufgabe von Erziehung darin gesehen, Menschen als urteilsfähig anzusprechen, sie im Üben ihrer Urteilskraft zu unterstützen und ihnen zu helfen, ein eigenes Urteil über sich und die Welt in ihren Erscheinungsformen zu fällen. Dies gilt auch für das Aufwachsen 
im rechtlichen Sinne: Heranwachsende können ihre Rechte nur dann einfordern, wenn sie gelehrt und gelernt werden. Ein Kind kann im pädagogischen Verständnis nur durch die Zuteilung von Rechten zur Rechtsperson werden, eine reine Statuszuschreibung reicht nicht aus. Lern- und Erfahrungsmöglichkeiten, von denen auch tatsächlich Gebrauch gemacht werden kann, sind deshalb in der Erziehung unabdingbar und die Bereitstellung dieser Möglichkeiten in der Verantwortung und Pflicht eines jeden Erwachsenen, der pädagogisch reflektiert das Aufwachsen eines Kindes unterstützen will.

\section{9. "Exemplar-Sein«}

Im Hinblick auf die von uns im Zusammenhang mit Bucks Überlegungen aufgeworfene Frage nach dem Status des Heranwachsenden, wäre das Miteinanderumgehen in der Erziehung damit so zu gestalten, dass der sich entwickelnde Mensch sein Verständnis von »Exemplar-Sein « auch in den Dimensionen des Rechts entwickeln und in die Kommunikation einzubringen vermag. Ohne die Anerkennung der Bildsamkeit des heranwachsenden Menschen gibt Erziehung als individuell zu verantwortende und zu gestaltende Begleitung des Lebenslaufs von Kindern und Jugendlichen generell wenig Sinn, ohne die Beachtung der rechtlichen Dimension des Miteinanderumgehens in der gesellschaftlichen Öffentlichkeit würde sie zudem lückenhaft sein.

Im Sinne eines zukünftigen Erwachsenen sitzt der heranwachsende Mensch in der Erziehung - metaphorisch gesprochen - mit am Tisch und hat das Recht, sich an seiner Erziehung aktiv beteiligen zu können. Weil das Kind erziehungsbedürftig und -fähig ist und bereits eine gemeinsame Erziehungsgeschichte begonnen wurde, hat es dieses (moralische)
Recht. Die Gleichzeitigkeit des Kindes als "educandus " und als "Person aus eigenem Recht « schließen sich dabei weder aus, noch stehen sie im Widerspruch zueinander. Im Sinne der von Buck beschriebenen "Methodenlehre der moralischen Bildung" sind Moral und Recht vielmehr aufeinander zu beziehen, um ein Urteil über die "Thunlichkeit und Unthunlichkeit“ des Handelns fällen zu können. Denn das Sittengesetz hängt nicht im luftleeren Raum, sondern ist eine Orientierungsgröße, die in ihrer Einbettung in konkrete Formen des Miteinanderumgehens realistisch beurteilt werden sollte.

Hinzu kommt, dass sich "das Kind " in einem modernen Erziehungsverständnis nicht mehr als schutzbedürftiges, passives und auf Hilfe von Erwachsenen angewiesenes Wesen darstellen lässt - diese Sicht ist zu einseitig. Gerade weil das Kind in seinem Lernen nicht vertretbar und damit in diesem Sinne unhintergehbar ist, kann die erwachsene Person für eine gelingende Erziehung nicht ausschließlich eine paternalistische Vertretungsfunktion übernehmen. Wäre dies möglich, wüsste sie bereits, was das einzelne Kind in der jeweiligen Situation für seine Entwicklung und sein Aufwachsen braucht und brauchen wird und würde sie keine Fehler machen können. Der Erzieher wäre allwissend und das Kind bzw. der Jugendliche nicht mehr als das Objekt seiner Bearbeitung. ${ }^{46}$ Will man aber die Reduktion auf den bloßen Objektstatus vermeiden, muss dem heranwachsenden Menschen in der Erziehung der Status zugesprochen werden, selbst als Original von Urteilen über die "Thunlichkeit und Unthunlichkeit von Handlungen « in Erscheinung zu treten. Der Erzieher ist damit herausgefordert, eine Form des Miteinanderumgehens mitzugestalten, in der sich der heranwachsende Mensch als Subjekt seiner moralischen Bildung einbringen kann - und zwar in jeder Phase seines Aufwachsens. Die Ordnung 
des Erzieherischen ist insofern in ihrer Dynamik und Kommunikabilität auszuweisen. Jedes starre Schema scheitert an dieser Aufgabe. Andererseits scheint es aber auch nicht ausreichend zu sein, wenn man die Dynamiken und Kommunikationsformen lediglich aus der distanzierten Position eines externen Beobachters erforscht. Von dieser Warte aus ist es wenig aussagekräftig, wenn die ,Nähe، der Pädagogik zum erzieherischen Geschehen abgelehnt wird. Das ist zu einfach.

Die Allwissenheit des Erziehers ist nicht gegeben, die erwähnten Spannungen lassen sich nicht dauerhaft auflösen und als gesellschaftliche Daueranforderung ist Erziehung in jeder Generation von Unsicherheit, Komplexität und Unbestimmtheit geprägt. Dem Erzieher bleibt deshalb keine andere Möglichkeit, als den Erziehungssituationen in einer Haltung, die Kindern Achtung entgegenbringt und das einzelne Kind die eigene Würde erfahren lässt, mit Vertrauen, Zuversicht und Urteilsvermögen zu begegnen. Denn erhalten Kinder und Jugendliche keine Möglichkeit, sich als Akteure ihrer Entwicklung mit ihrer originären Perspektive auf die Welt einbringen und verhalten zu dürfen und zu können, droht diese zu verkümmern. ${ }^{47}$ Am Beispiel der rechtlichen Dimension des Miteinanderumgehens haben wir dies als Problem zu markieren versucht. Ein solches Problem bietet vielleicht das Potential, die ,Nähe، der Pädagogik und die Distanziertheit der Bildungsforschung aufeinander zu beziehen.

\section{Anmerkungen}

1 Auf der Suche nach Auskunft fällt auf, dass in vielen Handbüchern, Enzyklopädien und Lexika ein Lemma Exemplarität fehlt oder das Stichwort nicht im Register aufgeführt wird. Das gilt für folgende Werke aus den vergangenen 50 Jahren: Handbuch pädagogischer
Grundbegriffe. Hrsg. v. J. Speck u. G. Wehle. Studienausgabe. München: Kösel 1970; Handbuch philosophischer Grundbegriffe. Hrsg. v. H. Krings, H.M. Baumgartner u. Chr. Wild. Studienausgabe. München: Kösel 1973; Europäische Enzyklopädie zu Philosophie und Wissenschaften. Hrsg. v. H.J. Sandkühler. Hamburg: Felix Meiner 1990; Lexikon der philosophischen Begriffe. Hrsg. v. A. Ulfig. Wiesbaden: Fourier 1997; Erziehungswissenschaft. Ein Grundkurs. Hrsg. v. D. Lenzen. 5. Aufl. Reinbek bei Hamburg: Rowohlt 2002; Enzyklopädie Philosophie und Wissenschaftstheorie. Hrsg. v. J. Mittelstraß. Sonderausgabe. 4 Bde. Stuttgart, Weimar: J.B. Metzler 2004; Wörterbuch der phänomenologischen Begriffe. Hrsg. v. H. Vetter. Hamburg: Felix Meiner 2004. - Dort wo ein Stichwort im semantischen Raum von Exemplarität vorhanden ist, erfährt man selten etwas Nennenswertes: Das Wörterbuch der philosophischen Begriffe. Hrsg. v. A. Regenbogen u. U. Meyer. Hamburg: Felix Meiner 1998, S. 209 bietet lediglich eine Übersetzung von 'Exemplar und 'exemplarisch ‘. Im Pädagogik-Lexikon. Hrsg. v. G. Reinhold, G. Pollak u. H. Heim. München, Wien: R. Oldenbourg 1999, S. 191-194 äußert sich Wolfgang Klafki auf dreieinhalb Seiten im Eintrag 'Exemplarisches Lehren und Lernen, Exemplarisches Prinzip،. Wer eine ausführlichere Auskunft sucht, wird fündig im Historischen Wörterbuch der Philosophie. Hrsg. v. J. Ritter. Bd. 2: D-F. Sonderausgabe. Darmstadt: Wissenschaftliche Buchgesellschaft 2019, Sp. 820-823, wo Günther Buck über 'Beispiel, Exempel, exemplarisch، schreibt.

2 Berck, Karl-Heinz: Biologieunterricht - exemplarisch für Exemplarisches. In: Zeitschrift für Didaktik der Naturwissenschaften 2 (1996) 17-24, S. 23. Berck stellt hier eine Vermutung in den Raum und keine Feststellung.

3 Vor allem sexemplarisch، ist ein oft verwendetes Wort in wissenschaftlichen Publikationen. Im Handbuch Wissenschaftssoziologie. Hrsg. v. S. Maasen et al. Wiesbaden: Springer Fachmedien 2014 z.B. wird das Wort sexemplarisch mehr als 15mal verwendet, aber nicht einmal selbst zum Thema gemacht. Dies mag in einem Handbuch für Wissenschaftssoziologie noch angehen, aber schwer nachvollziehbar dürfte es sein, wenn in einem im Auftrag einer Didaktikgesellschaft herausgegebenen Sammelband 63mal die Ausdrücke 
'Exemplarität، und sexemplarisch، verwendet werden, ohne dass an einer Stelle auf deren Bedeutung eingegangen wird (Naturwissenschaftliche Bildung als Grundlage für berufliche und gesellschaftliche Teilhabe. Hrsg. v. Chr. Maurer. Gesellschaft für Didaktik der Chemie und Physik Jahrestagung in Kiel 2018. Regensburg: Universität Regensburg 2019).

4 Wörterbuch der Pädagogik. Hrsg. v. W. Böhm u. S. Seichter. 17. Aufl. Paderborn: Ferdinand Schöning 2018, S. 155.

5 Es ist dann vielleicht auch wenig überraschend, wenn bei den Lehrern ebenfalls "Exemplarität kaum ein Thema ist“, wie die Forschung berichtet (Niermann, Anne: Professionswissen von Lehrern des Mathematikund Sachunterrichts. '... man muss schon von der Sache wissen. ' Paderborn: Julius Klinkhardt 2017, S. 193). Einen weiteren Grund vermutet Karl-Heinz Berck in der Organisation der Hochschulausbildung, wie er sie am Beispiel der Biologielehrerausbildung kennt: "Alle Lehrer sollten so ausgebildet werden, daß sie genügend biophilosophisches und wissenschaftstheoretisches Wissen besitzen, um fundamentale Themen überhaupt zu erkennen und dann auch zu unterrichten. Bei der immer geringer werdenden Unterrichtszeit an Hochschulen wohl eher ein vergeblicher Wunsch « (Berck 1996, S. 23).

6 Diesen "Grundgedanken" fasst Wolfgang Klafki folgendermaßen zusammen: "Bildendes Lernen, das die Selbständigkeit des Lernenden fördert, also zu weiterwirkenden Erkenntnissen, Fähigkeiten, Einstellungen führt, wird nicht durch reproduktive Übernahme von Einzelkenntnissen und -fertigkeiten gewonnen, sondern dadurch, daß sich der Lernende an einer begrenzten Zahl von ausgewählten, repräsentativen Beispielen (Exempeln) aktiv allgemeine Erkenntnisse, Fähigkeiten, Einstellungen, m.a.W.: das jeweils Wesentliche, Strukturelle, Typische, Grundlegende, Prinzipielle eines Problembereiches erarbeitet. Mit Hilfe solcher allgemeinen (’kategorialen`) Einsichten und Fähigkeiten werden dann jeweils Gruppen strukturgleicher oder ähnlich strukturierter Einzelphänomene und -probleme zugänglich bzw. lösbar « (Klafki, Wolfgang: Exemplarischer Ansatz. In: Neue Sammlung 26 (1986) 593-595, S. 593).

7 Künzli David, Christine/Franziska Bertschy/Antonietta Di Giulio: Bildung für eine
Nachhaltige Entwicklung im Vergleich mit Globalem Lernen und Umweltbildung. In: Schweizerische Zeitschrift für Bildungswissenschaften 32 (2010) 213-231, S. 219.

8 Bartsch, Silke: Subjektorientierung. Ein Beitrag zur kompetenzorientierten Aufgabengestaltung in der Verbraucherbildung. In: Haushalt in Bildung \& Forschung 1 (2012) 52-64, S. 57.

9 Waldmann, Michael R.: Kategorisierung und Wissenserwerb. In: Allgemeine Psychologie. Hrsg. v. J. Müsseler. 2., neu bearb. Aufl. Heidelberg: Spektrum Akademischer Verlag 2008, S. 377-427, S. 377.

10 Thomas Rucker hat kürzlich dargelegt, dass ein Unterricht nicht dazu in der Lage ist, "Schüler für ein Leben in Selbstbestimmung freizusetzen«, wenn er nicht "mit einer Aufforderung zum Entwurf eigener Werturteile verbunden“ wird. Dies kann, so Rucker, nur im Konzept eines erziehenden Unterrichts gedacht werden (Rucker, Thomas: Unterricht, Exemplarität und Subjektivität. In: Pädagogische Rundschau 74 (2020) 397-414, S. 409f.).

11 Lohrmann, Katrin et al.: Die Bedeutung der (Un-)Ähnlichkeit von Beispielen für den Aufbau von konzeptuellem Wissen. In: Zeitschrift für Grundschulforschung 7 (2014) 60-73, S. 61.

12 "Gemeinsam ist den verschiedenen nationalen Steuerungsstrategien [...] die Einführung von Bildungsstandards und standardbasierten Tests « (Thiel, Felicitas/Kai S. Cortina/ Hans Anand Pant: Steuerung im Bildungssystem im internationalen Vergleich. In: Das Selbstverständnis der Erziehungswissenschaft. Geschichte und Gegenwart. Hrsg. v. R. Fatke u. J. Oelkers. Zeitschrift für Pädagogik Beiheft 60. Weinheim u.a.: Beltz Juventa 2014, S. 123-138, S. 124). Diese Neuausrichtung hat zu einem grundlegenden Wandel, einem Kulturwandel, in den Bildungseinrichtungen geführt. Man denkt in "Modellvorhaben«, die auf "das Hauptziel der breiten Umsetzung zur Veränderung, d.h. Verbesserung, der Bildungslandschaft bezogen werden müssen « (Koch, Hans Konrad: Gute Beispiele verändern die Landschaft noch nicht! Referat zur Eröffnung der Fachtagung ,Aus guten Beispieln lernen des Forum Bildung. In: Aus guten Beispielen lernen. Fachtagung des Forums Bildung am 14. September 2001 in Berlin. Bonn: BLK 2002, S. 6-9, S. 7). 
13 Der locus classicus ist Wilhelm Flitners 'Das Selbstverständnis der Erziehungswissenschaft in der Gegenwart‘, 1957 erstmals publiziert. In diesem Text wird die engagierte Reflexion zum Programm der geisteswissenschaftlichen Pädagogik erhoben (heute in: Theoretische Schriften. Bd. 3: Abhandlungen zu normativen Aspekten und theoretischen Begründungen der Pädagogik. Besorgt u. mit einem Nachw. vers. v. U. Herrmann. Paderborn: Ferdinand Schöningh 1989, S. 310-349). Pädagogik als Wissenschaft im Verständnis Flitners ist ihrem "Ausgangspunkt nach [...] pragmatisch, insofern sie mit einem 'Engagement des Denkenden beginnt « (Flitner, Wilhelm: Pädagogik (1961). In: Theoretische Schriften. Bd. 3: Abhandlungen zu normativen Aspekten und theoretischen Begründungen der Pädagogik. Besorgt u. mit einem Nachw. vers. v. U. Herrmann. Paderborn: Ferdinand Schöningh 1989, S. 413-421, S. 420). Die Funktion pragmatischer Wissenschaften ist nach Flitner nicht smaterialistisch، zu verstehen, weil ihnen ein "Engagement für das Gute" eingeschrieben sei: "Ziel der medizinischen Forschung z. B. ist nicht, daß Ärzte und Apotheker Geld verdienen, und das Ziel der juristischen Forschung ist nicht, die Herrschaft der herrschenden Klasse oder Partei zu sichern; das hieße die Sache auf den Kopf stellen. Der Sinn dieser Disziplinen ist, platonisch gesprochen, ,das Gute; ihre Kenntnisse stehen im Dienst, ihre Einsichten sind sidealistisch،, indem sie in einer sldee،, einem ethischen Grundgedanken zentriert sind." Das Engagement sei auch dem pädagogischen Denken und Handeln immanent, weil es sich in Erziehung, Unterricht und Bildung um Sachverhalte handle, die ohne ethische Überlegungen nicht sinnvoll beurteilt werden könnten (Flitner, Wilhelm: Rückschau auf die Pädagogik in futuristischer Absicht (1976). In: Theoretische Schriften. Bd. 3: Abhandlungen zu normativen Aspekten und theoretischen Begründungen der Pädagogik. Besorgt u. mit einem Nachw. Vers. V. U. Herrmann. Paderborn: Ferdinand Schöningh 1989, S. 487-498, S. 493). Mit dieser Orientierung an einem "ethischen Gedankengang “ kann die Bildungsforschung bis heute nichts anfangen. Ihr gesamtes Instrumentarium ist auf einen Gegenstandsraum ausgerichtet, für den dieser "Gedankengang« von ihr nicht kultiviert wird. Umgekehrt hat die Geisteswissenschaftliche Pädagogik keinen "Gedankengang “ zu kultivieren vermocht, in dessen Gegenstandsraum das 'System Schule، eine bedeutende Rolle spielen würde.

14 Öhmann, Emil: Das Suffix-tät im Deutschen. In: Neuphilologische Mitteilungen 24 (1923) 157-164, S. 160.

15 Adelung, Johann Christoph: Grammatischkritisches Wörterbuch der hochdeutschen Mundart mit beständiger Vergleichung der übrigen Mundarten, besonders aber der Oberdeutschen. 2., vermehrte u. verb. Aufl. Leipzig: Johann Gottlob Immanuel Breitkopf und Compagnie 1793, S. $1986 f$.

16 Mellin, Georg Samuel Albert: Encyklopädisches Wörterbuch der Kritischen Philosophie oder Versuch einer fasslichen und vollständigen Erklärung der in Kants kritischen und dogmatischen Schriften enthaltenen Begriffe und Sätze mit Nachrichten Erläuterungen und Vergleichungen aus der Geschichte der Philosophie begleitet, und alphabetisch geordnet. 2. Bd. 1. Abt. Jena, Leipzig: Friedrich Frommann 1799, S. 465-466, S. 465f.

17 Kant, Immanuel: Kritik der Urteilskraft (1790). In: Kant's Werke. Hrsg. v. d. Königl. Preuß. Akademie d. Wissenschaften. Bd. V. Berlin: Georg Reimer 1913, S. 165-485, S. 308.

18 Mellin 1799, S. 466.

19 Kant 1790/1913, S. 232.

20 Buck, Günther: Kants Lehre vom Exempel. In: Archiv für Begriffsgeschichte. Bd. XI. Bonn: H. Bouvier u. Co. 1967, S. 148-183.

21 Kant, Immanuel: Kritik der praktischen Vernunft (1788). In: Kant's Werke. Hrsg. v. d. Königl. Preuß. Akademie d. Wissenschaften. Bd. V. Berlin: Georg Reimer 1913, S. 1-164, S. 30. Der kategorische Imperativ wird von Kant in weiteren Fassungen behandelt, die in der Grundlegung zur Metaphysik der Sitten (1785). In: Kant's Werke. Hrsg. v. d. Königl. Preußischen Akademie der Wissenschaften. Bd. IV. Berlin: Georg Reimer 1911, S. 385-464, S. 421-438 zu finden sind.

22 Hartmann, Nicolai: Kants Metaphysik der Sitten und die Ethik unserer Tage (1924). In: Kleinere Schriften III: Vom Neukantianismus zur Ontologie. Berlin: Walter de Gruyter \& Co. 1958, S. 339-345, S. 349.

23 Ebd., S. 346.

24 Buck 1967, S. 149.

25 Das wirft grundlegende Fragen auf: Gibt es, so könnte man fragen, eine srichtige، 
Relation zwischen einem spezifischen Allgemeinen und seinem Besonderen, die nur in bestimmten Beispielen dargestellt werden kann? Mit anderen Worten: Sind Beispiele nicht beliebig wählbar, sondern immer nur in Abhängigkeit von einer spezifischen Relation Allgemeines | Besonderes? Oder ist es vielmehr so, dass die Relation Allgemeines | Besonderes ,nur، eine Differenz ist, die beliebig zum Einsatz kommen kann? Ist, mit anderen Worten, alles in der Relation Allgemeines | Besonderes darstellbar?

26 Kant, Immanuel: Metaphysik der Sitten (1797). In: Kant's Werke. Hrsg. v. d. Königl. Preußischen Akademie d. Wissenschaften. Bd. VI. Berlin: Georg Reimer 1914, S. 203-493, S. 479.

27 Kant 1797/1914, S. 479. Anm.

28 Buck 1967, S. 151.

29 Ebd.

30 Ebd., S. 178.

31 Ebd., S. 182.

32 Ebd., S. 183.

33 Gabriel, Markus: Die Welt der Welten. In: Bonner Enzyklopädie der Globalität. Hrsg. v. L. Kühnhardt u. T. Mayer. Wiesbaden: Springer 2017, S. 53-64, S. 62.

34 Weizsäcker, Viktor von: Menschenführung. Nach ihren biologischen und metaphysischen Grundlagen betrachtet. 7. Aufl. Göttingen: Vandenhoeck \& Ruprecht 1955, S. 8.

$35 \mathrm{Zu}$ einer solchen Reduktion kommt es bspw. in der (neuro-)psychologischen Forschung, die das Lernen, Erinnern, Wahrnehmen usw. von Personen in einem Labor untersucht. Die Daten, die uns von diesen Forschungen dargeboten werden, bleiben insofern einseitig, als sie von sich aus nicht darzulegen vermögen, wie das, was eine Person, die an Elektroden angeschlossen wurde oder vor einem Bildschirm sitzt, tun oder unterlassen würde, wenn sie im Umgang mit ihresgleichen betrachtet würde. Die Aussage, dass im Labor erforschtes Lernen eine Relevanz aufweise für das Miteinanderumgehen, bleibt solange eine unbewiesene Behauptung, wie es der Forschung nicht gelingt, die Form des Miteinanderumgehens zu beschreiben, in die genau dieses Lernen integriert ist, und zwar so, dass es diese Form hervorbringt. Auf die Gefahr, die besteht, wenn durch methodische Reduktion gewonnene Befunde gleichwohl generalisiert werden, hat schon Karl Jaspers hingewiesen: "Behandelt man ein Einzelnes aus einem Ganzen, so schweben unausgesprochen eine oder mehrere Vorstellungen dieses Ganzen im Hintergrunde, oder die Behandlung bleibt verworren, widerspruchsvoll, undeutlich." (Jaspers, Karl: Psychologie der Weltanschauungen (1919). 4., unveränd. Aufl. Berlin, Göttingen, Heidelberg: Springer 1954, S. 18f.)

36 Arendt, Hannah: Adolf Eichmann. Von der Banalität des Bösen. In: Merkur 17 (1963) 759-776, S. 773. (Neu in: Die Botschaft des Merkur. Eine Anthologie aus fünfzig Jahren der Zeitschrift. Hrsg. v. K.H. Bohrer u. K. Scheel. Stuttgart: Klett-Cotta 1997, S. 152-169.) Arendt hat diese Überlegung von Jaspers, der eigentliche Ursprung ist aber bei Kant zu finden. In der Reflexion 903 seines Nachlasses beschreibt er, was der "egoist der Wissenschaft" benötigt, damit "dem gelehrten humanitaet« zukommt: "dass er seinen Gegenstand noch aus dem Gesichtspunkte anderer Menschen ansieht" (Kant, Immanuel: Reflexionen zur Anthropologie. In: Kant's Werke. Hrsg. v. d. Königl. Preußischen Akademie d. Wissenschaften. Bd. XV. Handschriftlicher Nachlass. Berlin: Walter de Gruyter 1923, S. 55-654, S. 394).

37 Anhalt, Elmar: Bildsamkeit - Erziehungsbedürftigkeit. In Handbuch Erziehung. Hrsg. v. U. Sandfuchs, W. Melzer, B. Dühlmeier u. A. Rausch. Bad Heilbrunn: Julius Klinkhardt 2012, S. 124-131, S. 127.

38 Kant, Immanuel 1785/1911, S. 436.

39 Ammann, Kira: Bildsamkeit und Kinderrechte. Ein kritisches Plädoyer aus erziehungswissenschaftlicher Perspektive. Weilerswist: Velbrück Verlag 2020, S. 262.

40 Das hier vorherrschende Verständnis von Kinderrechten geht über die grund- und menschenrechtliche Dimension der Lebensphase Kindheit und die in der UN-Konvention über die Rechte des Kindes festgehaltenen Artikel hinaus. Unter Berücksichtigung der kindlichen Schutzbedürftigkeit und fehlenden Erfahrung im Verhältnis zu Erwachsenen sowie dem gleichzeitigen Streben nach Autonomie, Mitsprache und Mitbestimmung, wird darunter "die ethische und moralische Verpflichtung von Erwachsenen verstanden, Kindern und Jugendlichen gegenüber die Achtung aufzubringen, sich als Akteure [...] ihrer Entwicklung mit ihrer originären Perspektive auf die Welt ein bringen und verhalten zu dürfen und zu können, weil andernfalls die Gefahr 
besteht, dass diese originäre Perspektive zu verkümmern droht“ (Ammann 2020, S. 44f.).

41 Balzer, Nicole; Drerup, Johannes: Dualismen, Dichotomien und Dogmatik. Kritische Anmerkungen zur Debatte über die Begründung der Kinderrechte. In: Berliner Debatte Initial, 28 (2017), 59-71, S. 60.

42 Herbart, Johann Friedrich: Umriss pädagogischer Vorlesungen (1835). In: Sämtliche Werke in 19 Bänden hrsg. v. K. Kehrbach u. O. Flügel. 2. Neudruck der Ausgabe Langensalza. Band 10, S. 69-196. Aalen: Scientia 1989, S. 69.

43 Anhalt, Elmar: Über den Ort der Bildsamkeit und des Bildsamkeitsbegriffs in Johann
Friedrich Herbarts pädagogischem Denken«. In: Vierteljahrsschrift für wissenschaftliche Pädagogik, 76, 151-176, S. 167.

44 Ipfling, Heinz-Jürgen: Grundbegriffe der pädagogischen Fachsprache. Begriffe zweisprachig deutsch-englisch. München: Ehrenwirth 1974, S. 48.

45 Herbart, Johann Friedrich: Über Erziehung unter öffentlicher Mitwirkung (1810). In: Sämtliche Werke in 19 Bänden hrsg. v. K. Kehrbach u. O. Flügel. 2. Neudruck der Ausgabe Langensalza. Band 3, S. 73-82. Aalen: Scientia 1989, S. 77.

46 Ammann 2020, S. 267.

47 Ebd. 